\title{
Constraining proto-planetary disc evolution using accretion rate and disc mass measurements: the usefulness of the dimensionless accretion parameter
}

\author{
Giovanni P. Rosotti, ${ }^{1 \star}$ Cathie J. Clarke, ${ }^{1}$ Carlo F. Manara,${ }^{2}$ Stefano Facchini ${ }^{3}$ \\ ${ }^{1}$ Institute of Astronomy, University of Cambridge, Madingley Road, Cambridge, CB3 OHA, UK \\ ${ }^{2}$ Scientific Support Office, Directorate of Science, European Space Research and Technology Centre (ESA/ESTEC), \\ Keplerlaan 1, 2201 AZ, Noordwijk, The Netherlands \\ ${ }^{3}$ Max-Planck-Institut für Extraterrestrische Physik, Giessenbachstrasse 1, 85748 Garching, Germany
}

Accepted 2017 March 08. Received 2017 March 04; in original form 2016 July 22

\begin{abstract}
We explore how measurements of protoplanetary disc masses and accretion rates provided by surveys of star forming regions can be analysed via the dimensionless accretion parameter, which we define as the product of the accretion rate and stellar age divided by the disc mass. By extending and generalising the study of Jones et al (2012), we demonstrate that this parameter should be less than or of order unity for a wide range of evolutionary scenarios, rising above unity only during the final stages of outside in clearing by external photoevaporation. We use this result to assess the reliability of disc mass estimates derived from $\mathrm{CO}$ isotopologues and submm continuum emission by examining the distribution of accretion efficiencies in regions which are not subject to external photoevaporation. We find that while dust based mass estimates produce results compatible with theoretical expectations assuming canonical dust/gas ratio, the systematically lower CO based estimates yield accretion efficiencies significantly above unity in contrast with the theory. This finding provides additional evidence that $\mathrm{CO}$ based disc masses are an under-estimate, in line with arguments that have been made on the basis of chemical modelling of relatively small samples. On the other hand, we demonstrate that dust based mass estimates are sufficiently accurate to reveal distinctly higher accretion efficiencies in the Trapezium cluster, where this result is expected given the evident importance of external photoevaporation. We therefore propose the dimensionless accretion parameter as a new diagnostic of external photoevaporation in other star forming regions.
\end{abstract}

Key words: accretion, accretion discs - protoplanetary discs - stars: variables: $\mathrm{T}$ Tauri, Herbig Ae/Be - stars: pre-main-sequence

\section{INTRODUCTION}

One of the main scientific motivations for the construction of facilities that can conduct surveys of large samples of protoplanetary discs is that the statistical distributions of disc properties can be used to place constraints on evolutionary models. In turn, these are needed to characterise the formation environments of planets and build a successful planet formation theory. This applies par excellence to observatories such as ALMA where disc surveys in submm continuum and emission lines from the isotopologues of CO (Ansdell et al. 2016; Barenfeld et al. 2016; Pascucci et al. 2016) yield

^ E-mail: rosotti@ast.cam.ac.uk estimates of disc masses (Williams \& Best 2014; Miotello et al. 2016b; Boneberg et al. 2016); in parallel, the possibility of simultaneous spectroscopic characterisation across a wide range of near ultraviolet to near infrared wavelengths with instruments such as X-shooter provides an opportunity to assemble data on disc accretion rates of unprecedented accuracy (Manara et al. 2016a; Alcalá et al. 2014, 2016; see also Herczeg \& Hillenbrand 2008; Ingleby et al. 2013).

There are however several inter-woven difficulties in straightforwardly using such data for constraining disc evolution models. There is no single family of disc evolution models that can be 'calibrated' against observational data: the models contain a number of uncertainties concerning the mechanism for angular momentum removal/redistribution 
and its associated timescales (Turner et al. 2014), combined with further uncertainties associated with the roles of internal/external photoevaporation (Alexander et al. 2014). At the same time, also, observational data is in need of calibration because of uncertainties in relating observed quantities to disc properties. This problem is particularly acute for dust and $\mathrm{CO}$ isotopologues because of their varying abundance with respects to the dominant mass component (Favre et al. 2013; McClure et al. 2016).

At first sight then, the problem of ill constrained theoretical models combined with uncertain observational calibration limits the utility of observational survey data. In this paper we present an analysis path which assesses the available data in relation both to its intrinsic reliability and to its ability to discern environmental differences between different star forming regions. Following Jones et al. (2012), the crux of the argument is based on comparing disc masses with the product of accretion rate and age. We assess the much larger observational dataset currently available and also generalise the theoretical models considered. For ease of discussion we introduce some new terminology, defining the dimensionless accretion parameter as

$\eta=\tau \dot{M} / M_{\text {disc }}$

and comparing observational estimates of this quantity with model predictions. In this definition a system with high dimensionless accretion parameter is one for which the accretion rate is high given its age and disc mass. We will show that self-regulation of disc evolutionary models implies that a high dimensionless accretion parameter cannot be achieved by simply invoking more rapid angular momentum transport within discs and show that it instead points to the role of external photoevaporation.

In outline we consider the properties of the whole family of possible theoretical models and use this to argue that the data in the literature generically favours the use of sub-mm dust continuum data (rather than CO isotopologue data) as a measure of disc mass. With this assumption we then further examine whether the existing data is accurate enough to find possible differences between star forming regions. In particular we consider the central region of the Orion Nebula Cluster (ONC) where external photo-evaporation is expected to be important and demonstrate that indeed, by comparison with low-mass star forming regions, the available data bears an imprint of the role of external photoevaporation in setting disc properties.

This paper is structured as follows. In section 2 we present theoretical arguments to illustrate the utility of the dimensionless accretion parameter previously mentioned. In Section 3 we analyse the existing data in light of the dimensionless accretion parameter we have introduced. Finally in Section 4 we draw our conclusions.

\section{THEORETICAL EXPECTATIONS}

\subsection{Viscous evolution}

We here consider discs that evolve as a result of what we loosely term viscous processes, i.e. processes that redistribute angular momentum within the disc, leading to radial (accretion) flows of velocity $v_{r}(r)$. Such processes are commonly invoked to explain the empirical evidence that young stellar objects accrete. Although the exact mechanism responsible for their existence is not currently known, the best candidate is the Magneto Rotational Instability (MRI); see Turner et al. (2014) for a review.

In the absence of other processes, viscous processes attempt to set up a quasi-steady state in which the local accretion rate $\dot{M}(r)$ is independent of radius over much of the disc. Defining the local viscous timescale as $t_{v}(r)=r / v_{r}$, and writing $\dot{M}(r)=2 \pi \Sigma r v_{r}$ (where $\Sigma$ is the local surface density in the disc) we can then write $\dot{M}(r)=2 \pi \Sigma r^{2} / t_{v}(r)$. Parametrising $\Sigma$ as a radial power law $\Sigma \propto r^{-p}$, where observationally $p$ is measured in the range 0.5 to 1.5 (Williams \& Cieza 2011), we conclude that $\Sigma r^{2}$ is an increasing function of $r$. Thus, if observed discs have been significantly shaped by viscous evolution, $t_{v}$ is an increasing function ${ }^{1}$ of $r$. In this case, the quasi-steady state has two additional properties:

(i) the outer disc radius is set by the requirement that $t_{v}$ in the outer disc is of order the system age $\tau$ (i.e. the disc has just had time to grow by viscous evolution to its present size);

(ii) since most of the disc mass is at large radii, $M_{\mathrm{disc}}(r) \sim$ $\pi \Sigma(r) r^{2}$ and the quasi-steady state condition can be written in the form $\dot{M}=\sigma M_{\mathrm{disc}}(r) / t_{v}(r)$, where $\sigma$ is a constant of order unity which depends on the surface density profile in the quasi-steady state.

Taken together, the two properties imply that the viscous quasi-steady state is characterised by the condition $\dot{M} \sim$ $M_{\text {disc }} / \tau$, i.e. $\eta=\tau \dot{M} / M_{\text {disc }} \sim 1$. We thus assign a dimensionless accretion parameter of unity to a disc that is accreting according to this expectation. Note how this establishes a connection between the mass accretion rate onto the star and the outer parts of the disc (typically at hundreds of au) where most of the mass resides.

The above qualitative description is borne out by many numerical calculations including those of Jones et al. (2012) and those presented below; it can also be demonstrated analytically in the case of discs with a simple power law parametrisation of viscosity on radius, which evolve according to viscous similarity solutions (Lynden-Bell \& Pringle 1974; Hartmann et al. 1998). It is however worth emphasising that the features noted above (in particular the prediction $\eta \sim 1$ ) are not dependent on this restrictive assumption and are a generic feature of discs that have attained a viscous quasi-steady state. We perform a series of numerical experiments to demonstrate this in Section 2.3 through using some extreme parameter choices for the viscous properties of the disc. In Section 2.4 we investigate instead what happens when a viscous quasi steady-state cannot be attained.

\subsection{Method}

We solve the evolution equation of the disc surface density $\Sigma(R, t)$ (e.g. Lynden-Bell \& Pringle 1974; Pringle 1981) due to the kinematical viscosity $v(\Sigma, R)$ :

$\frac{\partial \Sigma}{\partial t}=\frac{1}{R} \frac{\partial}{\partial R}\left[3 R^{1 / 2} \frac{\partial}{\partial R}\left(v \Sigma R^{1 / 2}\right)\right]-\dot{\Sigma}(R)$,

1 Note that this is equivalent to requiring that most of the disc mass is at large radii. 
where $\dot{\Sigma}(R)$ represents a potential loss-term to model the effects of external photo-evaporation.

The equation is discretised on a grid of points uniformly spaced in $R^{1 / 2}$ and then integrated in time using a standard explicit finite-difference method second order accurate in space and first order accurate in time (Pringle et al. 1986). When taking into account external photo-evaporation, we follow Clarke (2007) by removing mass from the outer edge of the disc. Namely, we locate the outer edge of the disc by finding the grid cell where the column density is closest to some low constant value (provided that this value is sufficiently low, the results are insensitive to the exact value due to the steepness of the surface density profile close to the outer edge; in our calculations we employ a value of $10^{-8} \mathrm{~g} \mathrm{~cm}^{-2}$ ). We then progress inwards removing a total mass of $\dot{M}_{\mathrm{ph}} \Delta t$, where $\Delta t$ is the length of the timestep and $\dot{M}_{\text {ph }}$ the total mass-loss due to external photo-evaporation, which is assumed (cf. Adams et al. 2004; Facchini et al. 2016) to be concentrated at the disc outer edge. Other works (Mitchell \& Stewart 2010; Anderson et al. 2013; Kalyaan et al. 2015) have taken a similar approach. See Clarke (2007) for the photo-evaporation rates that we assume, which are parametrised from Adams et al. (2004) and Johnstone et al. (1998) in the super-critical regime. They correspond to a value of the interstellar FUV field $G_{0}=3000$ (Habing 1968) for a $1 M_{\odot}$ star.

\subsection{Sensitivity of results to radial dependence of the viscosity}

In what follows we use as initial conditions a self-similar solution (Lynden-Bell \& Pringle 1974) with $\gamma=1$, an initial radius of $10 \mathrm{au}$ and an initial disc mass of $0.05 M_{\odot}$. The value of $\alpha$ (Shakura \& Sunyaev 1973) is 0.01 (if not specified otherwise) and we assume a flaring disc with $H / R=0.03$ at $1 \mathrm{au}$.

In this section we present some numerical experiments which have the goal of illustrating the claims made previously. In particular, we show that $\eta \sim 1$ is reached even if there are radial variations in the efficiency of viscous transport. In Figure 1 we illustrate the highly artificial case that the viscosity is subject to large amplitude radial fluctuations superposed on a background law $v \propto r$ (left panel; the blue solid line is the background and the green dashed the oscillatory profile). The right hand panel illustrates that there is very little difference in the evolution of the dimensionless accretion parameter as a function of time: both models settle into the state where $\eta \sim 1$ once the system age exceeds the maximum viscous timescale in the disc (which is at large radii in both cases). Note that for $\gamma=1, \sigma$ equals 0.5 (see Section 2.1), which explains why the accretion parameter remains slightly smaller than unity.

In Figure 2 we show a more realistic case where the viscosity is dropped by a factor $f$ at radii between 1 and 10 $\mathrm{au}$, possibly corresponding to the case of a region of weak magneto-turbulence, a so-called dead zone (see Armitage 2011; Turner et al. 2014 for reviews of the topic). We test values of $f$ ranging from 1 (that is, no dead zone) to 1000 . In this case, the maximum viscous timescale in the disc is initially at the outer edge of the dead zone and is indicated by the three vertical dashed lines in Figure 2 (note that the case of $f=1000$ is outside the scale of the plot). Although the disc expands to the point that the viscous timescale at the outer edge is similar to $\tau$, the system cannot enter a quasi-steady state until $\tau$ exceeds this maximum value. The inclusion of a dead zone thus has the effect of merely delaying the onset of the quasi-steady state. Depending on the chosen parameters, the delay might be long enough that the disc enters into a quasi-steady state on a timescale longer than the observationally constrained lifetime of discs. In this case, we would expect the disc to accrete throughout its life with a dimensionless accretion parameter below unity.

\subsection{External photo-evaporation and other processes}

Several factors may however prevent or modify the attainment of a viscous quasi-steady state. First of all, depending on the nature of angular momentum transport, there may be no surface density profile that allows the condition that $\dot{M}$ is independent of $r$, for example if there are regions where the angular momentum transport is completely inhibited. In this case the system will undergo a series of accretion bursts due to gravo-thermal instabilities (Armitage et al. 2001); it would then spend the majority of its time in a state of low accretion where $\eta \ll 1$ (Jones et al. 2012). Secondly, disc evolution may be subject to other mass and angular momentum sinks. Recent work (e.g., Suzuki \& Inutsuka 2009; Bai \& Stone 2013; Fromang et al. 2013; Simon et al. 2013) has suggested that disc secular evolution may be strongly influenced by magnetically driven winds. The consequences of such winds for disc secular evolution however depend on the mass loss profile and, in particular, on how the ratio of the specific angular momentum removed by the wind to the local Keplerian value (the so-called dimensionless lever arm of the wind) depends on radius. Currently these radial dependences are poorly constrained (Armitage et al. 2013; Bai 2016) and we do not explore this scenario further here. On the other hand, disc thermal photoevaporation is reasonably well understood, whether it derives from internal or external radiation sources (Clarke et al. 2001; Owen et al. 2010; Johnstone et al. 1998; Adams et al. 2004; Clarke 2007; Anderson et al. 2013; Facchini et al. 2016) and in this case angular momentum and mass are removed in a ratio that is given by the local Keplerian specific angular momentum. Jones et al. (2012) demonstrated that internal photoevaporation (which drives inside-out clearing) results in $\eta<1$ (i.e. inhibits accretion). We now turn to investigate the effect of external photo-evaporation.

\subsubsection{External photo-evaporation}

In Figure 3 we show the case of a disc subject to external photoevaporation (light blue solid and green dotted dashed lines), compared with a control simulation without photoevaporation (orange dashed line). The chosen parameters are such that the disc initially expands viscously as normal (light blue part of the time evolution), but then reaches a radius where the external mass loss rate is comparable with the accretion rate (Clarke 2007; Anderson et al. 2013). At this point, the disc is cleared from the outside in (green part of the time evolution); the mass of the disc quickly decreases as the dominant mass reservoir in the disc (at large 

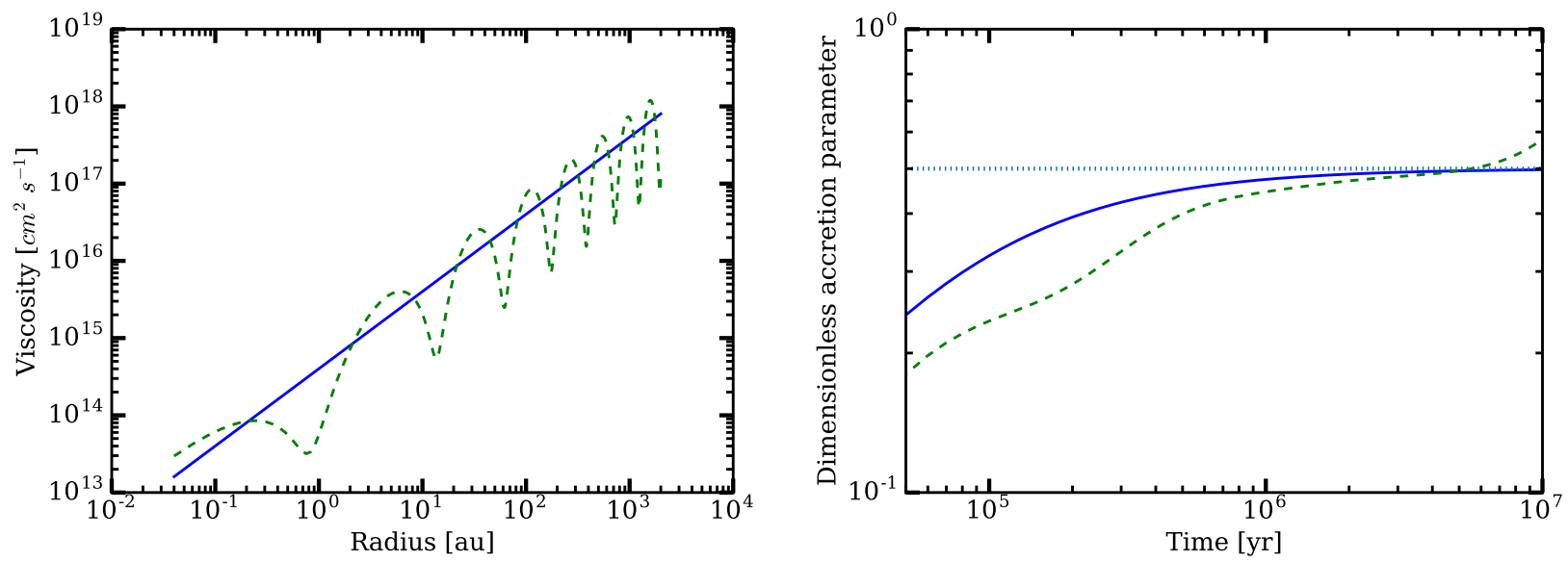

Figure 1. Left panel: the green dashed line shows the viscosity profile applied; for reference we plot also the background law $v \propto r$ (blue solid line). Right panel: evolution in time of the dimensionless accretion parameter for the two cases (the colors have the same meaning of the previous plot). For reference the horizontal blue dotted line shows a dimensionless accretion parameter of 0.5 . Despite the extreme substructure we artificially imposed on the viscosity, there is very little difference in the evolution of the dimensionless accretion parameter.

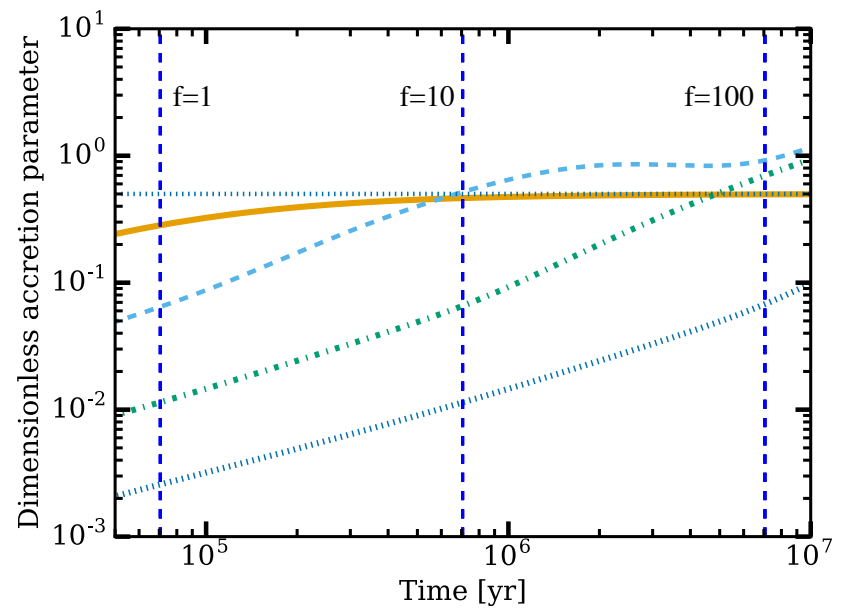

Figure 2. Evolution in time of the dimensionless accretion parameter for the case of a dead zone between 1 and $10 \mathrm{au}$. The different lines correspond to different values of the parameter $f$ (see text): 1 (orange solid), 10 (light blue dashed), 100 (green dotted-dashed) and 1000 (blue dotted). The dashed blue vertical lines represent the viscous timescale at the outer edge of the dead zone (i.e. $10 \mathrm{au}$ ) for the different values of $\mathrm{f}$ (note that the case of $f=1000$ is outside the scale of the plot). The blue dotted horizontal line shows a reference dimensionless accretion parameter of 0.5 . For $\mathrm{f}=1$ and 10 the dimensionless accretion parameter is not significantly altered. For $\mathrm{f}=100$ the dimensionless accretion parameter reaches unity only after several Myr; finally for $\mathrm{f}=1000$ the dimensionless accretion parameter is always lower than unity.

radii) is eroded. As the disc shrinks, the viscous time at the outer edge of the disc decreases because $t_{v}$ is an increasing function of radius (see Section 2.1). This leads to a progressive increase in $\eta$ during the clearing phase. We expect other processes that truncate the disc, for example dynamical encounters, to produce a similar effect. In the case of a dynamical encounter, however, the disc will revert back to a dimensionless accretion parameter of unity on a viscous time-scale. Summarising, the evolution is distinct in two phases, one characterised by $\eta \sim 1$ where the effects of external photo-evaporation are almost negligible, and one characterised by $\eta \gg 1$ during the outside in clearing.

In Figure 4, the initial conditions involve instead a case where external photoevaporation is important in controlling the disc radius from the beginning and hence $\eta$ increases monotonically throughout the evolution. In this case the disc always accretes efficiently.

We thus conclude that external photo-evaporation enhances the disc dimensionless accretion parameter above unity during the outside in dispersal phase (i.e. at the end of the disc lifetime), but that it is only markedly above unity during the very brief final clearing phases (see Figure 3).

\subsection{Summary of theoretical expectations}

Combining the results of our numerical experiments with those of Jones et al. (2012) we conclude that viscous evolution generally results in $\eta \lesssim 1$. The case $\eta \sim 1$ corresponds to the establishment of a viscous quasi-steady state; $\eta<1$ can result from the system age being younger than the maximum viscous timescale in the disc, from the fact that the viscosity prescription does not admit a quasi-steady state solution (episodic accretion ${ }^{2}$ ) or else from internal photoevaporation. The only scenario for which $\eta \gg 1$ is expected is that of external photoevaporation during the final stages of disc dispersal ${ }^{3}$. We summarise these results in Figure 5.

2 While episodic accretion produces high accretion efficiencies during the accretion burst phase, the disc spends most of its time accreting with low efficiencies (Jones et al. 2012).

3 Dynamical encounters can have a similar effect as well as mentioned in Section 2.4.1, but in practice photoevaporation becomes more important at substantially lower densities (Scally \& Clarke 2001). 


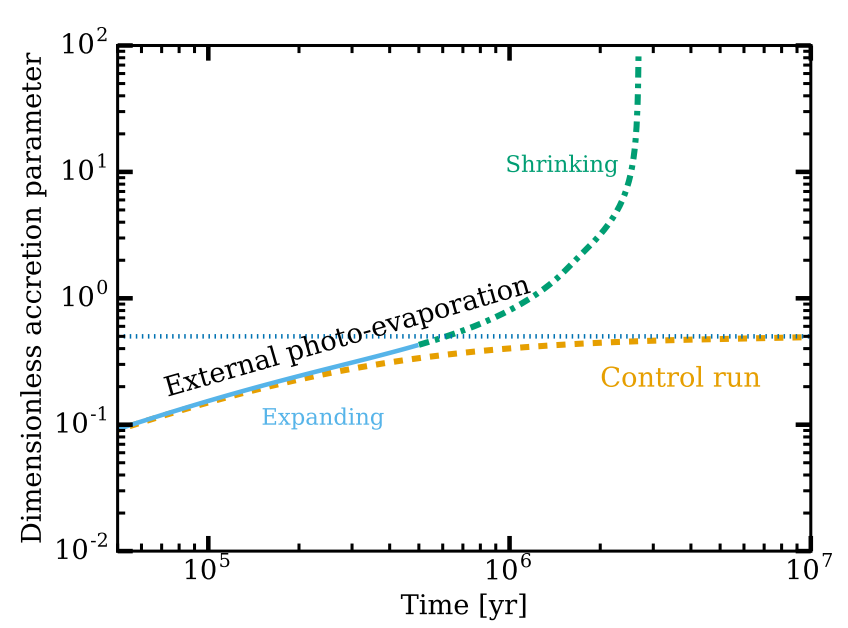

Figure 3. Dimensionless accretion parameter of an externally photo-evaporating disc. The light blue solid line marks the expanding phase of the disc and the green dotted dashed one the shrinking one. Shown on the plot (orange dashed line) there is also the result from a control run where we have not included external photo-evaporation and the reference dimensionless accretion parameter of 0.5 (horizontal blue dotted line). Notice how only in the shrinking phase external photoevaporation starts to matter; during this phase the dimensionless accretion parameter becomes noticeably higher than unity.

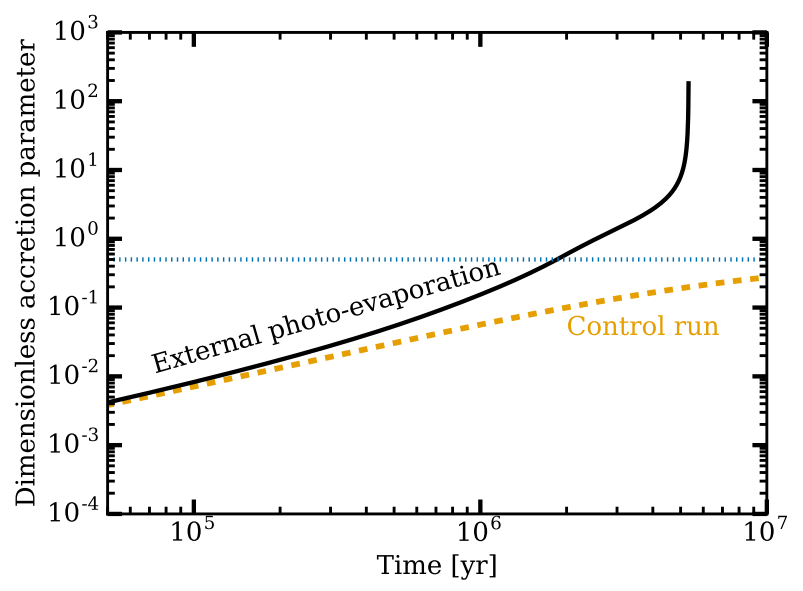

Figure 4. Same as Figure 3 but for a disc that only experiences the shrinking phase. Solid black line: photo-evaporating disc. Orange dashed line: control run without photo-evaporation. Horizontal blue dotted line: the reference dimensionless accretion parameter of 0.5 .

\section{COMPARISON WITH OBSERVATIONS}

In this section we gather existing data from the literature and analyse them in light of the theoretical considerations presented in the previous section. Note that, to compare to observations, we now plot accretion rate and disc mass, which are the quantities that the observations attempt to assess. Computing a dimensionless accretion parameter requires choosing an age of the system. In contrast to Jones

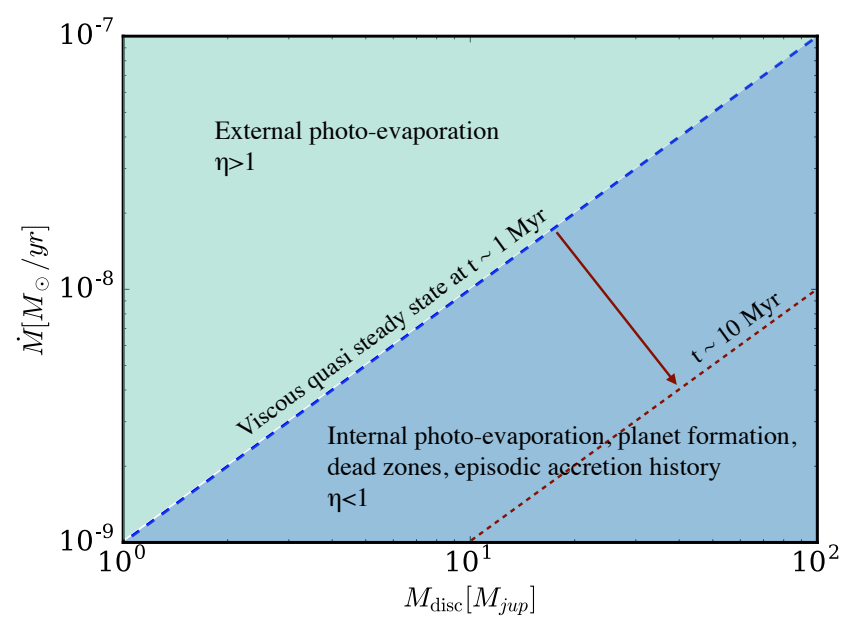

Figure 5. Cartoon showing how the $M / \dot{M}$ ratio can inform us on the processes governing disc evolution. To draw the different regions we have assumed an age of the system; for reference we used 1 Myr, which is the typical age of observed proto-planetary discs, and indicated on the plot how the regions would move for an age of $10 \mathrm{Myr}$

et al. (2012), in this work we do not use the individual ages of each star, as they are notoriously unreliable (e.g. Soderblom et al. 2014) but instead indicate in the plots the lines along which the dimensionless accretion parameter is unity for various assumptions about the stellar age. The typical ages of star forming regions containing proto-planetary discs are in the range 1-10 Myr (Fedele et al. 2010), although they might be underestimated by a factor of 2 according to recent estimates (Bell et al. 2013).

\subsection{The validity of the available tracers of the disc mass}

As mentioned in Section 1, observations do not directly yield the disc masses. By comparing the theoretical expectations with the observational data available from the surveys, we will examine how arguments developed in the previous section can be used to assess the reliability of the two main tracers of disc mass.

\subsubsection{Disc masses obtained via CO measurements}

Thanks to recent improvements in sensitivity to sub-mm emission lines, it is now possible to assess gas masses through the observation of the rare, faint $\mathrm{CO}$ isotopologue $\mathrm{C}^{18} \mathrm{O}$. Thermo-chemical modelling (e.g., Miotello et al. 2016b) is necessary because the abundance relative to molecular hydrogen depends on photo-dissociation and freeze-out of the molecule. We collected data from the Lupus survey (Ansdell et al. 2016 for the masses and Manara et al. (2016b) for the accretion rates) and from the sample reported in Williams \& Best (2014) (mostly consisting of sources from Taurus). For the latter sample, we surveyed the literature to find accretion rates (see Table 1). We used the Siess et al. (2000) stellar evolutionary models in the interest of uniformity of calculation of accretion rates. 
Table 1. Values of the accretion rate collected from the literature for the sample of Williams \& Best (2014).

\begin{tabular}{|c|c|c|c|}
\hline Object & Disc mass $\left[M_{\odot}\right]$ & $\dot{M}\left[M_{\odot} \mathrm{yr}^{-1}\right]$ & Reference \\
\hline AA Tau & $1.5 \mathrm{e}-03$ & $2.3 \mathrm{e}-08$ & Ingleby et al. (2013) \\
\hline CI Tau & $3.0 \mathrm{e}-03$ & $4.2 \mathrm{e}-08$ & McClure et al. (2013) \\
\hline CY Tau & $1.0 \mathrm{e}-03$ & 7.3e-09 & Hartmann et al. (1998) \\
\hline DL Tau & $5.0 \mathrm{e}-04$ & $6.8 \mathrm{e}-08$ & White \& Ghez (2001) \\
\hline DO Tau & $2.0 \mathrm{e}-03$ & $9.6 \mathrm{e}-08$ & Hartmann et al. (1998) \\
\hline Haro 6-13 & $6.0 \mathrm{e}-03$ & $8.4 \mathrm{e}-08$ & Salyk et al. (2013) \\
\hline IQ Tau & $7.0 \mathrm{e}-04$ & $2.0 \mathrm{e}-08$ & Hartmann et al. (1998) \\
\hline TW Hydra & $5.0 \mathrm{e}-04$ & $1.2 \mathrm{e}-09$ & Manara et al. (2014) \\
\hline DM Tau & $9.0 \mathrm{e}-03$ & $8.3 \mathrm{e}-09$ & Manara et al. (2014) \\
\hline GG Tau & $1.3 \mathrm{e}-02$ & $9.7 \mathrm{e}-09$ & Hartmann et al. (1998) \\
\hline IM Lup & $2.5 \mathrm{e}-02$ & $7.8 \mathrm{e}-09$ & Alcalá et al. (2016) \\
\hline
\end{tabular}

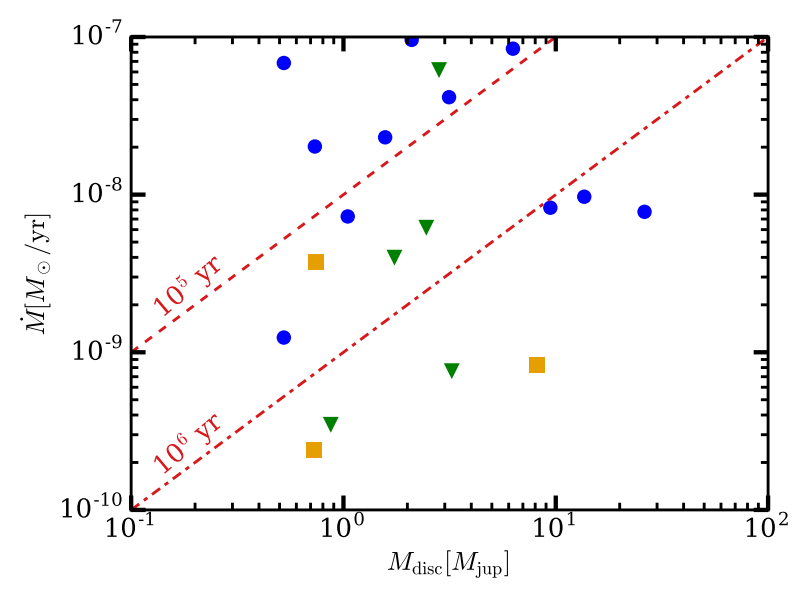

Figure 6. Measurements of accretion rate and disc masses (deduced from the gas) for all the sources where they are available. Blue points are for the sample of Williams \& Best (2014), green triangles for Lupus (Ansdell et al. 2016), orange squares for the objects with low accretion from the Lupus sample. The dashed and dotted dashed lines correspond to accretion efficiencies of unity assuming that the typical age is $10^{5}$ and $10^{6}$ years respectively.

The data points show a low value of the $M_{\text {disc }} / \dot{M}$ ratio (see Figure 6). In particular, 12 out of the 19 data-points have a $M_{\text {disc }} / \dot{M}$ ratio smaller than $10^{6}$ years, and 7 even smaller than $10^{5}$ years. For comparison, the age of Taurus is estimated to be 1-2 Myr (Briceño et al. 2002; Luhman et al. 2003). Note that in the plot we report only discs with $\mathrm{C}^{18} \mathrm{O}$ detections. The sample is thus biased towards large disc masses (i.e. bright discs), so that the ratio for the full sample could be even smaller. Moreover, if the recent revision of stellar ages by a factor of 2 (Bell et al. 2013) is correct, the problem would be even more serious.

Figure 6 demonstrates that the distribution of points in the $M_{\text {disc }}, \dot{M}$ plane shows considerable scatter and is incompatible with all objects having a single dimensionless accretion parameter. We have seen in Section 2 that there are a variety of effects that can result in a dimensionless accretion parameter of less than unity; thus the most troubling points are those with high accretion rates for which the dimensionless accretion parameter is much larger than unity for any reasonable stellar age estimate. In Section 2 we argued that a high dimensionless accretion parameter is only to be expected in discs affected by external photoevaporation. However, we find this explanation unlikely. Firstly, neither Taurus nor Lupus contains massive OB stars able to drive large photo-evaporative flows. Secondly, even if external photo-evaporation were eventually important for the evolution of these discs, as suggested by the recently revised photo-evaporation rates by Facchini et al. (2016), the arguments presented in Section 2.4.1 show that a dimensionless accretion parameter significantly greater than unity can be sustained only at the end of the disc lifetime, during the outside in dispersal. We would then be in the uncomfortable situation in which we are seeing these discs close to the end of their lifetime. This is unlikely to hold true in different star forming regions.

The explanation that we favour is that gas masses based on $\mathrm{CO}$ isotopologues represent a substantial under-estimate of the true disc mass. Similar interpretations have been proposed in the astro-chemical community. For example, a comparison of masses derived from the $\mathrm{CO}$ and HD molecules in the nearby disc TW Hya (Bergin et al. 2013) has led Favre et al. (2013) to conclude that CO is substantially less abundant (by a factor ranging $10^{-2}-0.3$ ) compared with what normally assumed. Kama et al. (2016) obtained a similar large under-abundance of both $\mathrm{C}$ and $\mathrm{O}$ in the same system by simultaneous fitting of emission lines of $\mathrm{HD}$ and different $\mathrm{C}$ and $\mathrm{O}$ bearing species. Miotello et al. (2016a), intepreting the relatively low $\mathrm{CO}$ based masses, suggested that carbon could be sequestered from $\mathrm{CO}$ to more complex molecules, or locked up in larger bodies. McClure et al. (2016), again from HD measurements, found that DM Tau shows a carbon depletion up to a factor of 5 and GM Aur up to two orders of magnitude.

Here we demonstrate, for a sample much larger than that for which HD measurements are available, that available accretion rate data strongly disfavours the use of $\mathrm{CO}$ isotopologues data for the estimation of disc mass.

\subsubsection{Disc masses obtained from the dust}

We now assess the reliability of disc masses based on dust continuum measurements. This method has been widely used since the seminal work of Beckwith et al. (1990) although it is widely recognised that there are uncertainties associated with the unknown dust to gas ratio, the disc temperature, the grain opacity and the effect of finite op- 


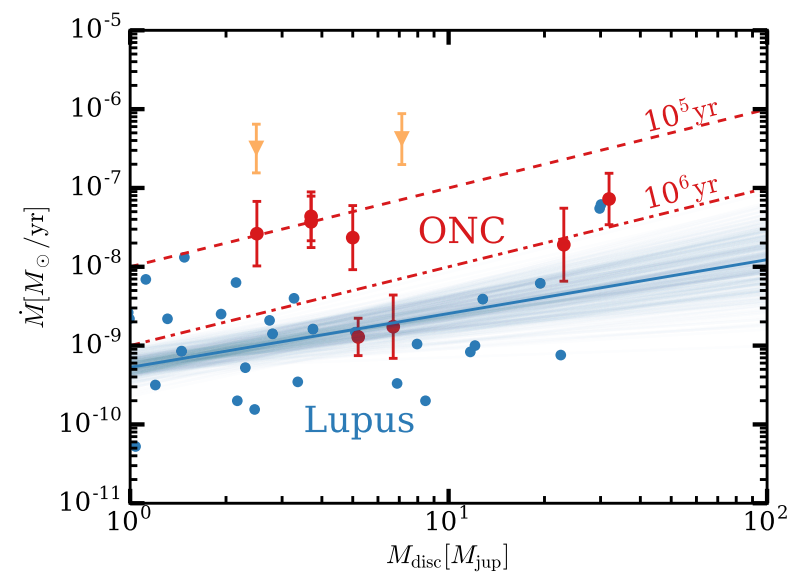

Figure 7. Measurements of accretion rate and disc masses (deduced from the dust) for objects in the Lupus cloud as blue points (Manara et al. 2016b). The blue solid line shows the best fit to the data, and in light blue we plot a subsample of the results of some Markov Monte-Carlo chains to show the uncertainty in the correlation. The objects in the ONC are shown as red points. The error bars take into account the error on the mass accretion rate propagated from the photometric error. The orange triangles denote objects that show some nebulosity in the HST images, for which the measurements of the accretion rate are more uncertain. Despite the relatively small size of the sample, it can be seen how the $M / \dot{M}$ ratio is smaller than the age of the system $(\sim 1 \mathrm{Myr}$, shown by the dotted dashed red line), which we interpret as due to the effects of external photo-evaporation.

tical depth. The blue points in Figure 7 replot part of the data presented in Manara et al. (2016b) for the Lupus region ${ }^{4}$ using dust derived masses. As noted by Manara et al. (2016b), there is a roughly linear correlation between dust mass and accretion rate with a relatively modest scatter; this implies a relatively narrow range of accretion efficiencies. Moreover the implied median dimensionless accretion parameter is unity for a plausible system age of $\sim 2.3 \mathrm{Myr}$, compatible with the age of the region (Alcalá et al. 2014).

It therefore appears that dust based mass estimates in Lupus $^{5}$ are broadly consistent with the theoretical expectations laid out in Section 2 and Jones et al. (2012). The relatively small scatter moreover suggests that discs have evolved into a viscous quasi-steady state. Note that this consistency is achieved assuming a canonical dust to gas ratio of 1:100, suggesting that the true value does not deviate from this by a large factor more than a factor $\sim 2$.

\subsection{Evidence of external photo-evaporation in Orion}

After having shown in 3.1.2 that dimensionless accretion parameter data in Lupus (using dust based mass estimates) is compatible with the generic expectations of evolutionary models, we need to further investigate whether dust based masses are sufficiently accurate for the dimensionless accretion parameter to be useful in diagnosing possible differences between disc populations. With this in mind we make a comparative study of dimensionless accretion parameter data in a low mass star forming region (Lupus) with that obtained in the Orion Nebula Cluster. We have abundant observational evidence for the importance of external photoevaporation in the ONC (e.g., Churchwell et al. 1987; Johnstone et al. 1998), notably in the form of the so-called proplyds (O'dell et al. 1993; Vicente \& Alves 2005): discs associated with an offset ionization front. They are commonly interpreted as discs losing mass through a wind driven by the FUV photons of $\theta_{1} \mathrm{C}$, the most massive star in the ONC; the ionization front traces the location where the wind becomes optically thin to EUV photons. Given the modelling in Section 2, we would thus expect accretion efficiencies to be significantly higher in the ONC since outside in-clearance reduces disc mass more than accretion rate. If this turns out to be the case, the dimensionless accretion parameter data can then be used as a diagnostic of the importance of external photoevaporation. This would enable the diagnostic to be used in future in analysing clusters where the proplyd population cannot be well characterised, either because of distance or because external photoevaporation drives a significative mass loss even if does not produce prominent proplyds.

We have collected dust masses in the ONC from the recent ALMA measurements of Mann et al. (2014) and Eisner et al. (2016). We then cross-correlated the catalogue with the measurements of accretion rate from $\mathrm{HST} \mathrm{H} \alpha$ photometry presented in Manara et al. (2012). This resulted in a sample of 11 objects with both $\dot{M}$ and disk mass measurements. Due to observational limitations, the sample is obviously not complete and is biased towards large disc masses and accretion rates, given that these sources are easier to detect. Figure 7 shows as red points the position of these targets on the $\dot{M}-M_{\text {dust }}$ plane, where $M_{\text {dust }}$ is the disc mass calculated assuming a gas-to-dust ratio of 100 . Despite the relatively small size of the sample, it can be seen how the $M / \dot{M}$ ratio is smaller than the age of the region $(\sim 2.2 \mathrm{Myr}$, Reggiani et al. 2011) in 8 out of 11 objects. To compare with Lupus, as mentioned in the previous section we have plotted with the blue line the best fit to the data in Lupus (Manara et al. 2016b) and in light blue a subsample of the results of some Markov Monte-Carlo chains to show the uncertainty in the correlation. It is clear how the distribution of efficiencies in the ONC is higher than in Lupus.

Some doubt could be cast on measurements of $\dot{M}$ de-

\footnotetext{
${ }^{4}$ We concentrate here only on the most massive discs for comparison with the ONC.

5 In the appendix we show that also for the sample of Williams \& Best (2014), used in the previous section, one recovers a dimensionless accretion parameter of roughly unity when using the masses derived from the dust, albeit with significantly more scattering than for Lupus sample.
} 
rived solely from $\mathrm{H} \alpha$ in proplyds, as contamination from unresolved ionization fronts of proplyds or from the strong background is possible and could lead to an overestimate of $\dot{M}$. We inspected visually the HST images for these objects and we did not find evidence for resolved ionization fronts, but for two objects we notice a small increase of the nebulosity of the background around them. We plot them as orange triangles and consider them as more uncertain; we note however that the other datapoints (red points) are still above the relation of Lupus (where instead the datapoints are scattered on both sides of the best fit, see the Lupus datapoints shown in blue) even if we were to remove them. As a further check, we have computed the median accretion timescale $M / \dot{M}$ of the blue points which is $\sim 3 \times 10^{5} \mathrm{yr}$, a factor of $\sim 7$ less than the age of the region.

We conclude that the inner region of the ONC is different from Lupus (Ansdell et al. 2016; Manara et al. 2016b). In light of the arguments presented in Section 2.4.1, this shows that the existing data is already of sufficient quality to recognise the fingerprint of external photo-evaporation in these discs. Indeed all of our sources (except one) lie inside a projected distance of $0.3 \mathrm{pc}$ from $\theta^{1}$ Ori $\mathrm{C}$, which is where external photo-evaporation is expected to be important (Störzer \& Hollenbach 1999). These discs are therefore currently being dispersed outside in, or, in other words, at the end of their life-time (see also discussion in Gorti et al. 2016).

\section{CONCLUSIONS}

In this paper we have shown how the availability of mass accretion rate and disc mass estimates for large samples of young stars provides an opportunity to both assess the reliability of these estimates and also to use them to explore systematic effects in different star forming regions.

We have introduced the concept of dimensionless accretion parameter ( $\eta$, see Eq. 1) as a diagnostic for distinguishing between various theoretical scenarios. Extending the work of Jones et al. (2012), we have demonstrated the mapping between the value of $\eta$ and a broad range of evolutionary scenarios (see Figure 5 for a schematic depiction). In particular we have confirmed that the dimensionless accretion parameter is expected to be around unity when disc evolution is driven by viscosity and the disc can reach a quasi steady-state. We have shown how this is a general result which does not depend on idealised assumptions about the viscosity. While a variety of processes can lower the dimensionless accretion parameter below unity, we have shown that external photo-evaporation is the only one capable of increasing it and that moreover a significant raising of dimensionless accretion parameter is only expected in advanced stages of outside-in clearing of discs.

The generic expectations from various evolutionary scenarios can also be used to assess the validity of the existing diagnostics of the disc mass. When considering disc masses derived from $\mathrm{C}^{18} \mathrm{O}$ in the weakly irradiated environments of Lupus and Taurus, we have shown how at face value the only way to interpret the current data is if the evolution of those discs would be dominated by external photo-evaporation. Because of the lack of massive stars in those regions, we reject this interpretation and propose that the current models used for interpreting CO data lead to substantial underestimates of the true disc gas mass, in line with similar findings based on detailed chemical modelling of small numbers of sources. Dust based gas estimates however result in accretion efficiencies of around unity as expected, suggesting that the dust can be employed as a reasonable measure of the disc mass, with a value of the dust to gas ratio close (within a factor of $\sim 2$ ) to the canonical ISM value of 100 .

To show that the existing data (using dust based disc mass estimates) is already accurate enough to find differences between regions through dimensionless accretion parameter analysis, we have also perfomed this analysis in the ONC using existing observational data. These discs are subject to an intense ultraviolet field and are thus expected to drive considerable photo-evaporative flows which, as shown in Section 2, should result in $\eta$ values considerably greater than unity. The available data suggests that this is indeed the case (Figure 7). The sample is however currently small and biased towards objects with large disc masses and accretion rates. Further studies are required to substantiate the effect in larger samples.

We also highlight that, if confirmed, it implies that dimensionless accretion parameter data can be used as a new diagnostic of the importance of environmental disc evaporation in star forming regions.

\section{ACKNOWLEDGEMENTS}

We thanks an anonymous referee for comments that improved the clarity of the paper. This work has been supported by the DISCSIM project, grant agreement 341137 funded by the European Research Council under ERC-2013ADG. CFM gratefully acknowledges an ESA Research Fellowship. We thank Phil Armitage, Jacob Simon, Tilman Birnstiel, Mario Flock, Leonardo Testi and Antonella Natta for inspiring discussions and Richard Booth and Mihkel Kama for providing comments on a draft version of this paper.

\section{REFERENCES}

Adams F. C., Hollenbach D., Laughlin G., Gorti U., 2004, ApJ, 611,360

Alcalá J. M., et al., 2014, A\&A, 561, A2

Alcalá J. M., et al., 2016, preprint, (arXiv:1612.07054)

Alexander R., Pascucci I., Andrews S., Armitage P., Cieza L., 2014, Protostars and Planets VI, pp 475-496

Anderson K. R., Adams F. C., Calvet N., 2013, ApJ, 774, 9

Ansdell M., et al., 2016, ApJ, 828, 46

Armitage P. J., 2011, ARA\&A, 49, 195

Armitage P. J., Livio M., Pringle J. E., 2001, MNRAS, 324, 705

Armitage P. J., Simon J. B., Martin R. G., 2013, ApJ, 778, L14

Bai X.-N., 2016, ApJ, 821, 80

Bai X.-N., Stone J. M., 2013, ApJ, 769, 76

Barenfeld S. A., Carpenter J. M., Ricci L., Isella A., 2016, ApJ, 827,142

Beckwith S. V. W., Sargent A. I., Chini R. S., Guesten R., 1990, AJ, 99, 924

Bell C. P. M., Naylor T., Mayne N. J., Jeffries R. D., Littlefair S. P., 2013, MNRAS, 434, 806

Bergin E. A., et al., 2013, Nature, 493, 644 
Boneberg D. M., Panić O., Haworth T. J., Clarke C. J., Min M., 2016, MNRAS, 461, 385

Briceño C., Luhman K. L., Hartmann L., Stauffer J. R., Kirkpatrick J. D., 2002, ApJ, 580, 317

Churchwell E., Felli M., Wood D. O. S., Massi M., 1987, ApJ, 321,516

Clarke C. J., 2007, MNRAS, 376, 1350

Clarke C. J., Gendrin A., Sotomayor M., 2001, MNRAS, 328, 485

Eisner J. A., Bally J. M., Ginsburg A., Sheehan P. D., 2016, ApJ, 826,16

Facchini S., Clarke C. J., Bisbas T. G., 2016, MNRAS, 457, 3593

Favre C., Cleeves L. I., Bergin E. A., Qi C., Blake G. A., 2013, ApJ, 776, L38

Fedele D., van den Ancker M. E., Henning T., Jayawardhana R., Oliveira J. M., 2010, A\&A, 510, A72

Fromang S., Latter H., Lesur G., Ogilvie G. I., 2013, A\&A, 552, A71

Gorti U., Liseau R., Sándor Z., Clarke C., 2016, Space Sci. Rev.,

Habing H. J., 1968, Bull. Astron. Inst. Netherlands, 19, 421

Hartmann L., Calvet N., Gullbring E., D’Alessio P., 1998, ApJ, 495,385

Herczeg G. J., Hillenbrand L. A., 2008, ApJ, 681, 594

Ingleby L., et al., 2013, ApJ, 767, 112

Johnstone D., Hollenbach D., Bally J., 1998, ApJ, 499, 758

Jones M. G., Pringle J. E., Alexander R. D., 2012, MNRAS, 419, 925

Kalyaan A., Desch S. J., Monga N., 2015, ApJ, 815, 112

Kama M., et al., 2016, A\&A, 592, A83

Luhman K. L., Stauffer J. R., Muench A. A., Rieke G. H., Lada E. A., Bouvier J., Lada C. J., 2003, ApJ, 593, 1093

Lynden-Bell D., Pringle J. E., 1974, MNRAS, 168, 603

Manara C. F., Robberto M., Da Rio N., Lodato G., Hillenbrand L. A., Stassun K. G., Soderblom D. R., 2012, ApJ, 755, 154

Manara C. F., Testi L., Natta A., Rosotti G., Benisty M., Ercolano B., Ricci L., 2014, A\&A, 568, A18

Manara C. F., Fedele D., Herczeg G. J., Teixeira P. S., 2016a, A\&A, 585, A136

Manara C. F., et al., 2016b, A\&A, 591, L3

Mann R. K., et al., 2014, ApJ, 784, 82

McClure M. K., et al., 2013, ApJ, 775, 114

McClure M. K., et al., 2016, ApJ, 831, 167

Miotello A., et al., 2016a, preprint, (arXiv:1612.01538)

Miotello A., van Dishoeck E. F., Kama M., Bruderer S., 2016b, A\&A, 594, A85

Mitchell T. R., Stewart G. R., 2010, ApJ, 722, 1115

O'dell C. R., Wen Z., Hu X., 1993, ApJ, 410, 696

Owen J. E., Ercolano B., Clarke C. J., Alexander R. D., 2010, MNRAS, 401, 1415

Pascucci I., et al., 2016, ApJ, 831, 125

Pringle J. E., 1981, ARA\&A, 19, 137

Pringle J. E., Verbunt F., Wade R. A., 1986, MNRAS, 221, 169

Reggiani M., Robberto M., Da Rio N., Meyer M. R., Soderblom D. R., Ricci L., 2011, A\&A, 534, A83

Salyk C., Herczeg G. J., Brown J. M., Blake G. A., Pontoppidan K. M., van Dishoeck E. F., 2013, ApJ, 769, 21

Scally A., Clarke C., 2001, MNRAS, 325, 449

Shakura N. I., Sunyaev R. A., 1973, A\&A, 24, 337

Siess L., Dufour E., Forestini M., 2000, A\&A, 358, 593

Simon J. B., Bai X.-N., Armitage P. J., Stone J. M., Beckwith K., 2013, ApJ, 775, 73

Soderblom D. R., Hillenbrand L. A., Jeffries R. D., Mamajek E. E., Naylor T., 2014, Protostars and Planets VI, pp 219241

Störzer H., Hollenbach D., 1999, ApJ, 515, 669

Suzuki T. K., Inutsuka S.-i., 2009, ApJ, 691, L49

Turner N. J., Fromang S., Gammie C., Klahr H., Lesur G., Wardle M., Bai X.-N., 2014, Protostars and Planets VI, pp 411-432

Vicente S. M., Alves J., 2005, A\&A, 441, 195

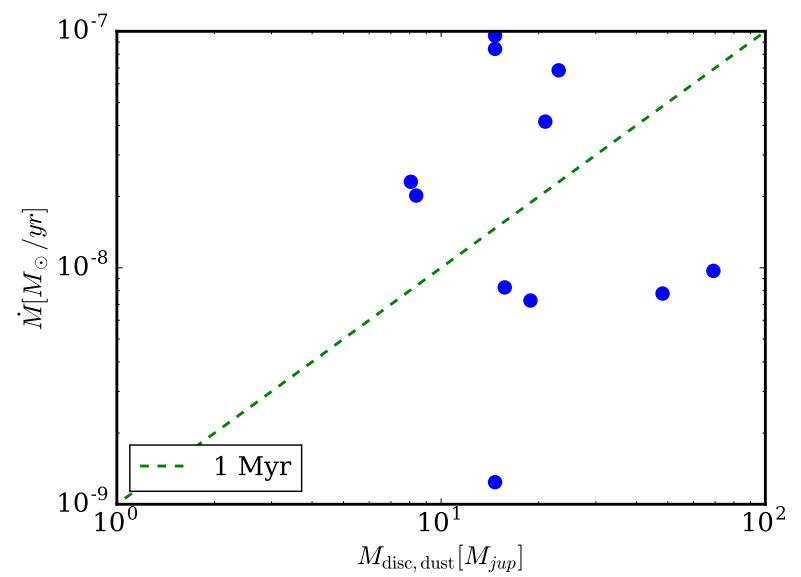

Figure A1. Measurements of disc masses (derived from the dust) and accretion rate for the sample of Williams \& Best (2014). The values are evenly spread around the $1 \mathrm{Myr}$ line.

White R. J., Ghez A. M., 2001, ApJ, 556, 265

Williams J. P., Best W. M. J., 2014, ApJ, 788, 59

Williams J. P., Cieza L. A., 2011, ARA\&A, 49, 67

\section{APPENDIX A: TAURUS DUST MASSES}

Manara et al. (2016b) have shown in Lupus that the dimensionless accretion parameter is close to unity using the disc masses derived from the dust and using a standard dust to gas ratio of 100 . Here we show that the same holds also for the sample of Williams \& Best (2014). We plot the results in Figure A1. The ratio of dust derived disc mass to accretion rate shows considerable scatter but is not systematically offset from the system age ( $\sim$ a Myr $)$. This indicates a dimensionless accretion parameter which is close to unity as found in Lupus. Note that the Lupus data have significant advantages compared to this sample: a higher number statistics, higher spectral resolution, better flux calibration and a homogeneous data analysis. This accounts for the much larger scatter in the Taurus data.

This paper has been typeset from a $\mathrm{T}_{\mathrm{E}} \mathrm{X} / \mathrm{LAT}_{\mathrm{E}} \mathrm{X}$ file prepared by the author. 